\title{
CORRECTION
}

\section{Correction to: Comments on the shape of voltammetric plots of reversible stoichiometric reactions for linear potential scan}

\author{
M. A. Vorotyntsev ${ }^{1} \cdot$ P. A. Zader ${ }^{1}$ D. V. Konev ${ }^{1,2}$
}

Published online: 27 November 2021

c) Springer-Verlag GmbH Germany, part of Springer Nature 2021

\section{Correction to: Journal of Solid State Electrochemistry} https://doi.org/10.1007/s10008-021-05073-y

The original article contained a mistake. Below the list of corrected formulas with original numeration is given:

$O^{0}(t) / R^{0}(t)=Z(t)$ for $x=0$ where $Z(t)=\exp \left[n F\left(E(t)-E^{0^{\prime}}\right) / R T\right]$

$D_{R} \partial R / \partial x=-D_{O} \partial O / \partial x=i / n F$ for $x=0$

$\partial R / \partial t=D_{R} \partial^{2} R / \partial x^{2}, \partial O / \partial t=D_{O} \partial^{2} O / \partial x^{2}$

for $0<x<\infty$

(6)

$R^{\prime}(x, t)=R^{*}-R(x, t), \partial R^{\prime} / \partial t=D_{R} \partial^{2} R^{\prime} / \partial x^{2}$,

$R^{\prime} \rightarrow 0$ for $t \rightarrow-\infty$ and for $x \rightarrow \infty$,

$R^{\prime}=R^{\prime}(0, t)=Z_{1 / 2}(t)\left[1+Z_{1 / 2}(t)\right]^{-1} R^{*}$ for $x=0$

$y=(n F / R T)\left(E-E_{1 / 2}\right)=(n F v / R T)\left(t-t_{1 / 2}\right)$

so that $Z_{1 / 2}=\exp (y), R^{\prime}(0, t)=R^{*}[1+\exp (y)]^{-1}$

(4) $i(t)=\left[(n F)^{3} v / R T\right]^{1 / 2} R^{*}\left(D_{R} / \pi\right)^{1 / 2} I(y,-\infty)$

where $I(y,-\infty) \equiv \int_{-\infty}^{y}\left(y-y^{\prime}\right)^{-1 / 2} \exp \left(y^{\prime}\right)\left[1+\exp \left(y^{\prime}\right)\right]^{-2} d y^{\prime}$

$R(x, t)=R^{*}, R^{\prime}(x, t) \equiv R^{*}-R(x, t)=0, O(x, t)=0$

for $t \leqslant t_{i}, 0<x<\infty$ and for $t<t_{i}, x=0$

(17)

$R^{0}\left(t_{i}+0\right) \equiv R\left(0, t_{i}+0\right)=\left[1+Z_{1 / 2}\left(t_{i}\right)\right]^{-1} R^{*}$,

$R^{\prime}\left(0, t_{i}+0\right) \equiv R^{*}-R^{0}\left(t_{i}+0\right)=Z_{1 / 2}\left(t_{i}\right)\left[1+Z_{1 / 2}\left(t_{i}\right)\right]^{-1} R^{*}$,

$O_{i}^{0} \equiv O^{0}\left(t_{i}\right)=Z\left(t_{i}\right) R_{i}^{0}$ for $t=t_{i}+0$

$i(t)=n F\left(D_{R} / \pi\right)^{1 / 2} \int_{t_{i}-0}^{t}\left(t-t^{\prime}\right)^{-1 / 2} \frac{d R^{\prime}\left(0, t^{\prime}\right)}{d t^{\prime}} d t^{\prime}$

(19)

$i(t)=n F\left(D_{R} / \pi\right)^{1 / 2}\left[\left(t-t_{i}\right)^{-1 / 2} R^{\prime}\left(0, t_{i}+0\right)+\int_{t_{i}+0}^{t}\left(t-t^{\prime}\right)^{-1 / 2} \frac{d R^{\prime}\left(0, t^{\prime}\right)}{d t^{\prime}} d t^{\prime}\right]$

$i(t)=\left[(n F)^{3} v / R T\right]^{1 / 2} R^{*}\left(D_{R} / \pi\right)^{1 / 2} I_{O C P}\left(y, y_{i}\right)$,

$I_{O C P}\left(y, y_{i}\right) \equiv\left(y-y_{i}\right)^{-1 / 2}\left[1+\exp \left(-y_{i}\right)\right]^{-1}+I\left(y, y_{i}\right)$,

where $I\left(y, y_{i}\right) \equiv \int_{y_{i}}^{y}\left(y-y^{\prime}\right)^{-1 / 2} \exp \left(y^{\prime}\right)\left[1+\exp \left(y^{\prime}\right)\right]^{-2} d y^{\prime}$

The original article can be found online at https://doi.org/10.1007/ s10008-021-05073-y.

M. A. Vorotyntsev

mivo2010@yandex.com

Frumkin Institute of Physical Chemistry

and Electrochemistry, Russian Academy of Sciences,

Moscow, Russia

2 Institute of Problems of Chemical Physics, Russian Academy

of Sciences, Chernogolovka, Russia 
$R^{* *}=\left[1+Z_{1 / 2}\left(t_{i}\right)\left(D_{O} / D_{R}\right)^{\chi}\right]^{-1} R^{*}$,

$O^{* *} / R^{* *}=Z\left(t_{i}\right)=\exp \left[n F\left(E_{i}-E^{0^{\prime}}\right) / R T\right]$

where $\chi=1 / 2-\lambda>0$

(25)

$R^{0}(t)=\left[1+Z_{1 / 2}\left(t_{i}\right)\right]\left[1+Z_{1 / 2}(t)\right]^{-1} \cdot R^{* *}=$

$=\left[1+Z_{1 / 2}\left(t_{i}\right)\right] \cdot\left[1+Z_{1 / 2}(t) \cdot\left(D_{O} / D_{R}\right)^{\chi}\right]^{-1} R^{*}\left[1+Z_{1 / 2}(t)\right]^{-1}$

(27)

$i(t)=n F\left(D_{R} / \pi\right)^{1 / 2} \int_{t_{i}-0}^{t}\left(t-t^{\prime}\right)^{-1 / 2} \frac{d R^{\prime}\left(0, t^{\prime}\right)}{d t^{\prime}} d t^{\prime}$

(28)

$i(t)=\left[(n F)^{3} v / R T\right]^{1 / 2} R^{*}\left(D_{R} / \pi\right)^{1 / 2} I_{E}\left(y, y_{i}\right)$

where $I_{E}\left(y, y_{i}\right)=\left[1+\exp \left(y_{i}\right)\right]\left[1+\exp \left(y_{i}\right)\left(D_{O} / D_{R}\right)^{\chi}\right]^{-1} I\left(y, y_{i}\right)$,

$I(y,-\infty) \cong I_{\text {appr }}(y,-\infty) \equiv \pi^{1 / 2} \exp (y)\left[1-2^{1 / 2} \exp (y)+\ldots\right]$ for $y<0,|y| \gg 1$

$I(y,-\infty) \cong I_{a p p r}(y,-\infty) \equiv y^{-1 / 2}\left(1+\pi^{2} / 8 y^{2}\right)$

for $y \gg 1$

(31)

$\Delta I_{O C P}\left(y, y_{i}\right) \equiv I(y,-\infty)-I_{O C P}\left(y, y_{i}\right)=$

$=-\left(y-y_{i}\right)^{-1 / 2}\left[1+\exp \left(-y_{i}\right)\right]^{-1}+\Delta I\left(y, y_{i}\right)$

where $\Delta I\left(y, y_{i}\right) \equiv I(y,-\infty)-I\left(y, y_{i}\right)=$

$=\int_{-\infty}^{y_{i}}\left(y-y^{\prime}\right)^{-1 / 2} \exp \left(y^{\prime}\right)\left[1+\exp \left(y^{\prime}\right)\right]^{-2} d y^{\prime}$
$\Delta I_{O C P}\left(y, y_{i}\right) \cong \Delta I_{O C P(\text { appr })}\left(y, y_{i}\right) \equiv$

$\equiv-\exp \left(y_{i}\right)\left[1 / 2\left(y-y_{i}\right)^{-3 / 2}-(3 / 4)\left(y-y_{i}\right)^{-5 / 2}+\ldots\right]$

for $y_{i}<0,\left|y_{i}\right| \gg 1, y-y_{i} \gg 1$

$\Delta I\left(y, y_{i}\right) \equiv I(y,-\infty)-I\left(y, y_{i}\right)=$

$=\int_{-\infty}^{y_{i}}\left(y-y^{\prime}\right)^{-1 / 2} \exp \left(y^{\prime}\right)\left[1+\exp \left(y^{\prime}\right)\right]^{-2} d y^{\prime}$

$\Delta I\left(y, y_{i}\right) \cong \Delta I_{a p p r}\left(y, y_{i}\right) \equiv$ $\equiv \exp \left(y_{i}\right)\left[\left(y-y_{i}\right)^{-1 / 2}-1 / 2\left(y-y_{i}\right)^{-3 / 2}+(3 / 4)\left(y-y_{i}\right)^{-5 / 2}+\ldots\right]$ for $y_{i}<0,\left|y_{i}\right| \gg 1, y-y_{i} \gg 1$

The original article has been corrected.

Publisher's Note Springer Nature remains neutral with regard to jurisdictional claims in published maps and institutional affiliations. 